\title{
Diferencias en los formantes vocálicos de [a] según el tipo de emoción y otras variables sociolingüísticas
}

\author{
Iñaki Gaminde Terraza* \\ Asier Romero Andonegi \\ Aintzane Etxebarria Lejarreta \\ Urtza Garay Ruíz \\ Universidad del País Vasco / Euskal Herriko Unibertsitatea \\ Grupo de Investigación EUDIA
}

\begin{abstract}
Resumen
El objetivo de este trabajo es analizar las diferencias observadas en las medidas del $\mathrm{fl}$ y $\mathrm{f} 2$ de la vocal [a] en un corpus de frases que expresan las emociones básicas alegría, tristeza y enfado producidas por hablantes bilingües precoces de vasco y español. Aunque existen numerosos trabajos que se ocupan de la descripción acústica de las emociones en español realizadas desde diferentes puntos de vista, creemos que nuestro trabajo aporta una nueva perspectiva, ya que incluye el fenómeno del bilingüismo y del contacto de lenguas, en el caso que nos ocupa, el español y el euskera. Además, teniendo en cuenta las características de nuestro corpus, hemos considerado
\end{abstract}

\footnotetext{
Para correspondencia, dirigirse a: Iñaki Gaminde Terraza (inaki.gaminde@ehu.es); Asier Romero Andonegi (a.romero@ehu.es); Aintzane Etxebarria Lejarreta (aintzane.etxebarria@, ehu.es) y Urtza Garay Ruíz (urtza.garay@ehu.es), Departamento de Didáctica de la Lengua y la Literatura, Escuela Universitaria de Magisterio de Bilbao, Universidad del País Vasco/ Euskal Herido Unibertsitatea, Barrio Sarriena s/n, 48940 Leioa (Bizkaia), España.
} 
interesante tener en cuenta otras variables además del tipo de emoción. Las variables investigadas son la lengua materna, la provincia de procedencia, el área sociolingüística de los informantes y el tipo de sílaba o, mejor dicho, el sintagma donde se ubica cada sílaba.

Palabras clave: análisis acústico, prosodia, emociones, bilingüismo.

THE IMPACT OF DIFFERENT TYPES OF EMOTIONS AND OTHER SOCIOLINGUISTIC VARIABLES ON THE F1 AND F2 FORMANT VALUES $\mathrm{OF}[\mathrm{A}]^{1}$

Abstract

The aim of this work is to analyze the differences observed in the measures of $\mathrm{fl}$ and $\mathrm{f} 2$ from the vowel [a] in a corpus of phrases that express the basic emotions happiness, sadness and anger produced by bilingual precocious speakers of Basque and Spanish. Though there exist numerous works that deal with the acoustic description of the emotions in Spanish realized from different points of view, we think that our work contributes a new perspective, since it includes the phenomenon of the bilingualism and of the contact of languages, in the case that occupies the Spanish and the Basque. In addition, taking into account the corpus type, we have considered to be interesting bear other variables in mind besides the type of emotion. The investigated variables are the mother language, the province of origin, the area sociolinguistics of the informants and the type of syllable, or rather the syntagm where every syllable is located.

Keywords: Acoustic analysis. Prosody. Emotions. Bilingualism.

Recibido: $12 / 03 / 15$

Aceptado: 31/07/15

\section{INTRODUCCIÓN}

El objetivo general de este trabajo es completar los datos expuestos en un trabajo anterior, realizado con el mismo corpus (Gaminde 2010). En aquel 
estudio presentábamos los resultados del análisis realizado con los datos de la frecuencia fundamental, la duración, la energía y la calidad de la voz. Además, también se compararon los resultados del español con los resultados de la lengua vasca.

Excluimos de aquel trabajo los datos aquí presentados, por no poderlos comparar sistemáticamente con los del euskera, y por la gran diversidad dialectal de esta lengua que hacía imposible en nuestro corpus disponer de las mismas vocales para todos los informantes.

Hemos creído interesante incluir en esta pequeña aportación los datos sobre el f1 y f2 de la vocal [a], así como el análisis de los mismos, ya que se trata de las vocales de un corpus amplio obtenido con hablantes bilingües precoces y recogido en todo el área de contacto entre ambas lenguas.

Junto con la presentación de los datos obtenidos, el objetivo específico es analizar las diferencias observadas en las medidas del $\mathrm{fl}$ y f2 de la vocal [a] en un corpus de frases que expresan las emociones básicas alegría, tristeza y enfado producidas por los mencionados hablantes bilingües precoces de vasco y español.

En numerosos trabajos se ha señalado la gran cantidad de factores, como la edad, el sexo, el estado de ánimo, etc., que afectan a los formantes de las vocales (Quilis 1988; Martínez Celdrán 1989; Gil 1990; Etxebarria 1990; Urrutia et alii 1995; Biemans 2000; Schötz 2006; Liscombe 2007; Gaminde 2010). Para el español se puede consultar el trabajo de síntesis de Bobadilla et alii (1999).

En el caso concreto de las emociones, se ha reseñado que las diferencias espectrales pueden contribuir en gran medida al reconocimiento de las emociones, así como que son las emociones las causantes de esas diferencias (Yildirim et alii 2004; Makarova y Petrushin 2007; Li et alii 2010; Feraru 2011; Pribil y Pribilová 2013). También existen algunos trabajos para la lengua vasca desde el campo de la síntesis del habla que corroboran estos hechos (Navas et alii 2006; Navas et alii 2007, Sainz et alii 2008).

Teniendo en cuenta las características de nuestro corpus, especificadas en la siguiente sección, hemos considerado interesante tener en cuenta otras variables además del tipo de emoción. 


\section{CORPUS Y METODOLOGÍA}

Para la confección del corpus se recogieron cuatro frases por cada informante, una neutra y las tres restantes una por cada emoción (alegría, tristeza y enfado). Además, en las frases elegidas se controló la sonoridad de todos los segmentos. Para recoger las frases, se solicitó a los informantes producir en primer lugar la frase neutra (1) y seguidamente emplear la misma frase, simulando cada una de las emociones solicitadas dentro de una situación hipotética de comunicación. La selección de esta frase está motivada por dos elementos: la sonoridad de todos los segmentos en la frase y la acentuación en sus grupos tonales.

\section{(1) La nena llevaba}

Todas las grabaciones se hicieron en el entorno natural de los informantes entre los años 2001 y 2007. Según la época de cada grabación, se han utilizado tres aparatos con micrófono externo: Sony minidics, Marantz PMD620 y Zoom Hn4. Una vez obtenidas las grabaciones para la transcripción, etiquetación y análisis de los mismos se ha utilizado el programa "Praat" (Boersma y Weenink 2009).

Una vez etiquetadas todas las señales, las medidas de los formantes se obtuvieron en el centro de cada vocal.

Para la recogida de los materiales, se contó con la colaboración de 50 informantes de las tres provincias del País Vasco, que son Álava/ Araba, Bizkaia, Gipuzkoa y Navarra ${ }^{2}$. Todas las informantes son mujeres con edades comprendidas entre 18 y 30 años, bilingües precoces y con estudios universitarios, cursados principalmente en euskera. Los estudios preuniversitarios de las informantes se realizaron en modelos bilingües. Además, se ha tenido en cuenta la distribución geográfica equilibrada entre las diferentes zonas (20 informantes en Bizkaia, 13 en Gipuzkoa, en 12 Navarra y en 5 Álava/Araba) y la lengua materna de las informantes. Por tanto, el 50\% tiene como lengua materna el vasco y ha adquirido el español como L2 dentro del sistema educativo (Grupo A), y el 50\% restante tiene como lengua materna el español y a su vez han adquirido el vasco como L2 (Grupo B). Para identificar a los informantes hemos utilizado el nombre de su

2 Se utiliza la nomenclatura oficial vasca para los nombres de las localidades. Ley 19/2011, de 5 de julio (BOE-A-2011-11606). 
localidad de origen. Los informantes encuestados, según su lengua materna, son los siguientes (en el mapa 1 se muestran las localidades encuestadas):

\section{Grupo A:}

Álava/Araba: Aramaio.

Bizkaia: Amorebieta-Etxano, Bermeo, Elorrio, Gernika-Lumo, Larrabetzu, Lekeitio; Lemoa, Mungia, Ondarroa y Zeanuri.

Gipuzkoa: Aizarnazabal, Andoain, Azkoitia, Donostia, Eibar, Errenteria, Ordizia, Orio, Oñati, Segura.

Navarra: Arruiz, Doneztebe-Santesteban, Elizondo, Leitza.

\section{Grupo B:}

Álava/Araba: Vitoria-Gasteiz1, Vitoria-Gasteiz2, Amurrio, Laudio.

Bizkaia: Balmaseda, Basauri, Bilbao1, Bilbao2, Carranza, Leioa, Lemoa, Portugalete, Ugao-Miraballes, Zamudio.

Gipuzkoa: Donostia, Elgoibar, Tolosa.

Navarra: Berasáin, Burlada, Pamplona1, Pamplona2, Sangüesa, Lumbier, Tudela1 y Tudela2.

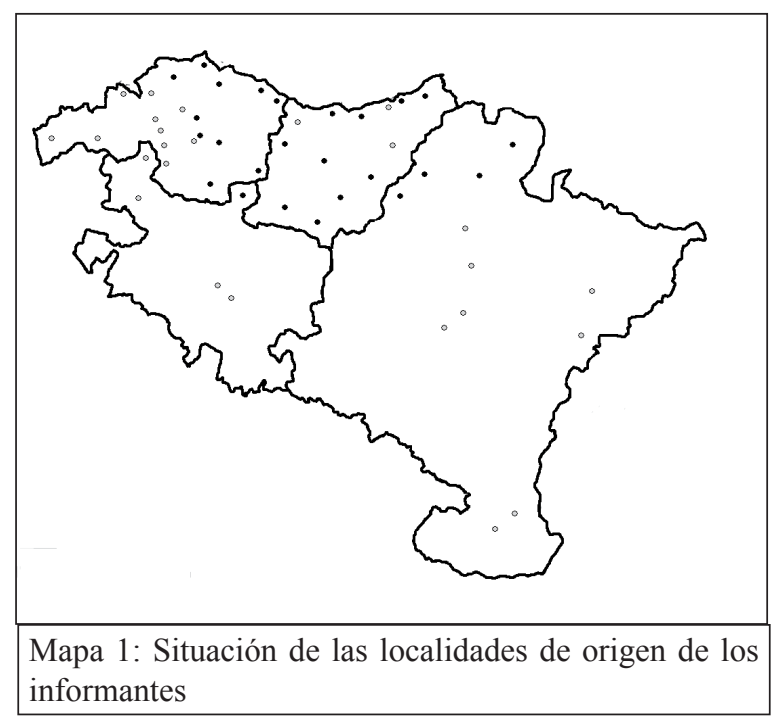

Por otra parte, hemos considerado interesante analizar la posible relación entre el origen geográfico de los informantes y los resultados obtenidos en las diferentes medidas. Para determinar las zonas de origen de los informantes, hemos tenido en cuenta dos criterios: por una parte, consideramos la división dialectal tradicional de la lengua vasca (Zuazo 1998) y, por otra parte, el 
conocimiento de la lengua que se da en las diferentes áreas (Soziolinguistika Klusterra 2013). De este modo, distinguimos las zonas en las que el conocimiento de la lengua vasca es superior al $50 \%$, de la población de aquellas localidades en el que el conocimiento es inferior a este porcentaje. Se han tenido en cuenta estas variables atendiendo a su influencia en los resultados de otras investigaciones, en las que el objetivo de análisis era también las emociones (Gaminde et al. 2013, 2014a, 2014b: Etxebarria et al. 2014).

Considerando ambos criterios, se puede dividir el territorio en siete zonas diferentes. Las zonas con menos del $50 \%$ de hablantes de la lengua vasca son: (1) la provincia de Álava, con la excepción del municipio de Aramaiona, (2) la zona occidental de Bizkaia, (3) la zona oriental de Gipuzkoa y (4) la zona media y meridional de Navarra. Las zonas con un porcentaje de hablantes del euskera superior al 50\% son: (5) la zona central oriental de la provincia de Bizkaia y la zona occidental de la provincia de Gipuzkoa, (6) la zona central de la provincia de Gizpuzkoa y (7) la zona norte de la provincia de Navarra. Los informantes se dividen de la siguiente manera en las zonas mencionadas (mapa 2):

(1): Amurrio, Vitoria-Gasteiz1, Vitoria-Gasteiz 2 y Llodio

(2): Balmaseda, Basauri, Bilbao1, Bilbao2, Carranza, Leioa, Portugalete, Ugao-Miraballes y Zamudio.

(3): Donostia (A), Donostia (B) y Errenteria

(4): Berasáin, Burlada, Lumbier, Pamplona1, Pamplona2, Tudela1, Tudela2, Sangüesa

(5): Aramaio, Bermeo, Elorrio, Gernika-Lumo, Larrabetzu, Lekeitio, Lemoa (A), Lemoa (B), Mungia, Ondarroa, Zeanuri, Amorebieta-Etxano, Eibar y Oñati

(6): Aizarnazabal, Andoain, Elgoibar, Ordizia, Orio, Segura, Tolosa

(7): Arruitz, Doneztebe-Santesteban, Elizondo y Leitza 


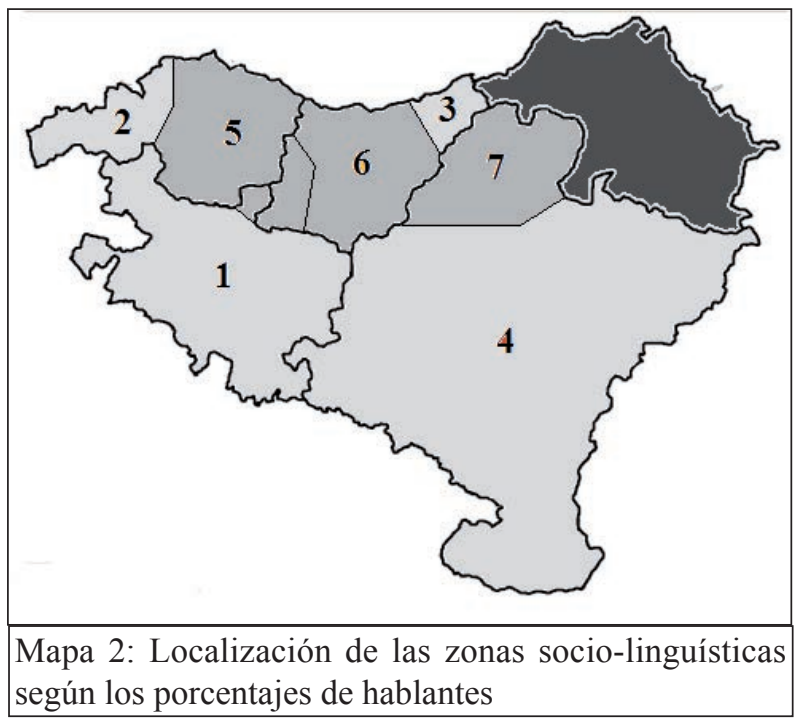

\section{ANÁLISIS DE LOS DATOS}

En este apartado presentamos el análisis de los datos obtenidos para el $\mathrm{f} 1$ y f2 de la vocal [a] según cada tipo de variable elegida. Para la realización del análisis de los datos, las variables elegidas son el tipo de sílaba (síl.) ${ }^{3}$, el tipo de emoción de la frase (emo.), la lengua materna de los informantes (l.m.), la provincia de origen (pro) y el área sociolingüística a la que pertenecen (á.soc.). Además, a este respecto hay que señalar que no se ha tenido en cuenta otro tipo de variables, como es el caso de los contextos de concurrencia de la vocal [a], ya que ambas vocales están precedidas o por consonantes sonantes [n] y [1], o por consonantes obstruyentes. En ambos casos, sin rasgos de realización espirantizada (Fernández Planas 2014).

La media total del f1 de las 600 vocales analizadas es $774,10 \mathrm{hz}$. con una desviación típica (Sd.) de 87,74. En cuanto al f2, la media es de 1611,56 hz. y una desviación de 263,53.

\footnotetext{
3 En este caso, la frase seleccionada tiene en todos sus casos un tipo silábico 2: CV, ya que en ningún caso se han elidido las aproximantes.
} 
En las siguientes líneas se presentan los datos de las variables analizadas.

Según el tipo de sílaba (tabla 1), la media más alta del f1 se da en la sílaba "la", seguida por "vá", "ba" y "na"; las diferencias no son estadísticamente significativas $(\mathrm{gl}=3 ; \mathrm{F}=1,431, \mathrm{p}=* 0,233)$. Con el $\mathrm{f} 2$ la media más alta se da en la sílaba "na", seguida de "la", "vá" y "ba"; en este caso las diferencias son estadísticamente significativas $(\mathrm{gl}=3, \mathrm{~F}=432,479, \mathrm{p}=0,000)$.

\begin{tabular}{|l|c|c|c|c|}
\hline & \multicolumn{2}{|c|}{$\mathbf{f 1}$} & \multicolumn{2}{c|}{$\mathbf{f 2}$} \\
\hline & Media & Sd & Media & Sd \\
\hline "la" & 785,85 & 85,14 & 1787,38 & 159,59 \\
\hline "na" & 765,75 & 99,36 & 1866,35 & 186,60 \\
\hline "vá" & 774,16 & 72,78 & 1423,60 & 114,72 \\
\hline "ba" & 770,65 & 91,15 & 1368,91 & 120,15 \\
\hline Tabla 1: Medias y desviaciones de f1 y f2 según el tipo de emoción \\
\hline
\end{tabular}

En el gráfico de dispersión 1, se puede ver claramente que las vocales correspondientes a las sílabas del sintagma anterior al verbo se sitúan en un espacio definido, frente al que ocupan las vocales de las sílabas del verbo. Las vocales de las sílabas del sintagma son más anteriores que las otras, mientras que las diferencias en la apertura no son importantes.

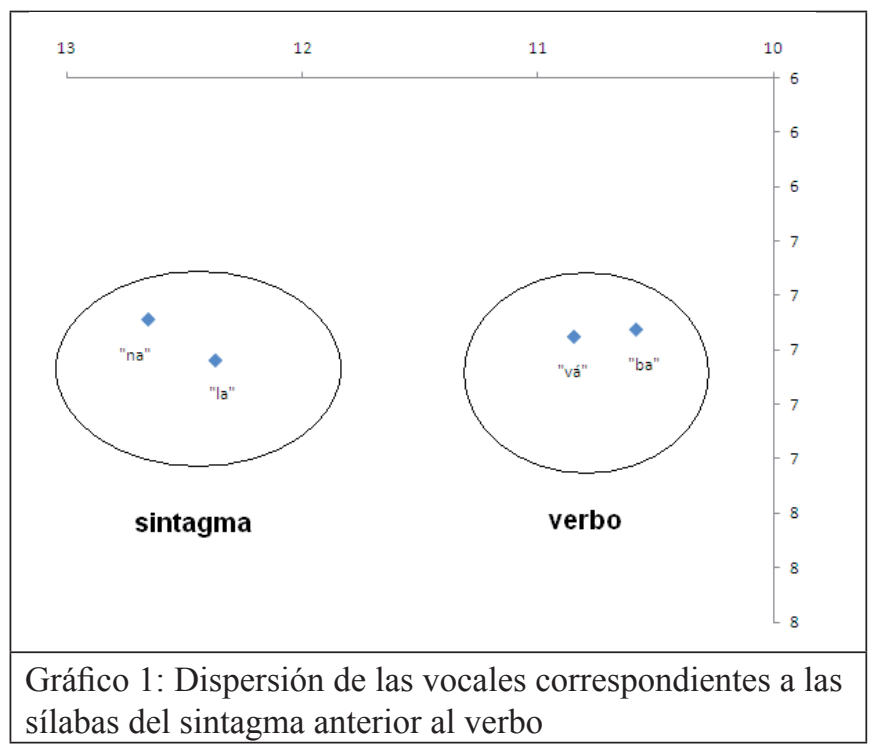


Según el tipo de emoción de la frase (tabla 2), la media del f1 es más alta con la alegría, seguida del enfado y la tristeza; aunque, como se muestra en la tabla, la diferencia entre la alegría y el enfado es muy escasa. De todas maneras, las diferencias son estadísticamente significativas $(\mathrm{gl}=2, \mathrm{~F}$ $=12,785, \mathrm{p}=0,000)$. En el $\mathrm{f} 2$ la media más alta se da con la alegría, seguida del enfado y la tristeza, las diferencias en este caso no son estadísticamente significativas $(\mathrm{gl}=2, \mathrm{~F}=1,544, \mathrm{p}=* 0,214)$.

\begin{tabular}{|l|c|c|c|c|}
\hline & \multicolumn{2}{|c|}{$\mathbf{f 1}$} & \multicolumn{2}{c|}{ f2 } \\
\hline & Media & Sd & Media & Sd \\
\hline Alegría & 787,57 & 86,15 & 1631,02 & 267,70 \\
\hline Tristeza & 749,00 & 82,13 & 1585,98 & 253,59 \\
\hline Enfado & 785,74 & 89,74 & 1617,69 & 268,33 \\
\hline \multicolumn{2}{|l}{ Tabla 2: Medias y desviaciones de f1 y f2 según el tipo de emoción de la frase } \\
\hline
\end{tabular}

En el gráfico de dispersión 2, se ve claramente que la tristeza es más alta y posterior que el enfado y la alegría. Por el contrario, las diferencias entre enfado y alegría son muy pequeñas.

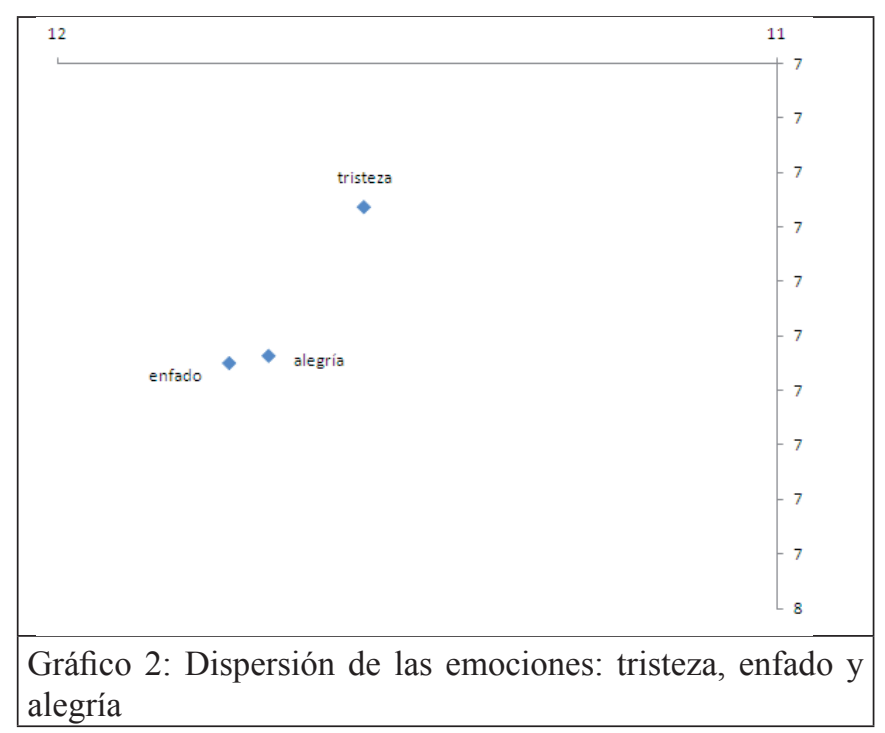


En la tabla 3, mostramos los datos de los formantes según las variables emoción de la frase y tipo de sílaba.

\begin{tabular}{|l|l|c|c|c|c|}
\hline \multicolumn{2}{|c|}{} & \multicolumn{2}{|c|}{ f1 } & \multicolumn{2}{c|}{ f2 } \\
\hline Emo & Síl & Media & Sd. & Media & Sd. \\
\hline \multirow{4}{*}{ alegría } & "la" & 788,00 & 85,94 & 1797,16 & 157,85 \\
\cline { 2 - 6 } & "na" & 785,76 & 103,27 & 1904,62 & 186,06 \\
\cline { 2 - 6 } & "Vá" & 778,58 & 70,38 & 1427,98 & 121,39 \\
\cline { 2 - 6 } & "ba" & 797,94 & 83,30 & 1394,30 & 113,93 \\
\hline \multirow{4}{*}{ tristeza } & "la" & 759,48 & 74,27 & 1761,02 & 158,60 \\
\cline { 2 - 6 } & "na" & 736,36 & 89,78 & 1814,50 & 193,93 \\
\cline { 2 - 6 } & "Vá" & 754,70 & 66,92 & 1419,32 & 116,26 \\
\cline { 2 - 6 } & "ba" & 745,46 & 94,98 & 1349,08 & 121,36 \\
\hline \multirow{4}{*}{ enfado } & "la" & 810,08 & 88,48 & 1803,96 & 162,13 \\
\cline { 2 - 6 } & "na" & 775,12 & 99,56 & 1879,94 & 170,95 \\
\cline { 2 - 6 } & "vá" & 789,20 & 77,69 & 1423,50 & 108,30 \\
\cline { 2 - 6 } & "ba" & 768,56 & 88,84 & 1363,36 & 122,89 \\
\hline
\end{tabular}

Tabla 3: Medias y desviaciones de cada formante según la emoción y tipo de sílaba

Como puede verse en el gráfico de dispersión 3, cada sintagma forma su propio espacio claramente definido y dentro de estos elementos, también cada sílaba.

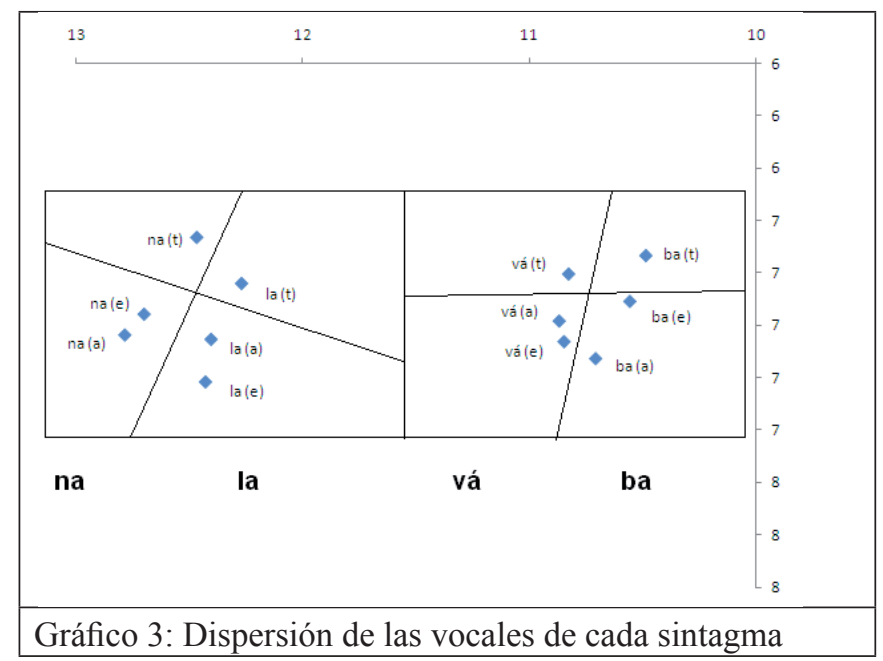


En la tabla 4, se muestran los datos del f1 y del f2 según la lengua materna de los informantes. En cuanto al f1, es ligeramente más alto para los informantes del grupo B que para los del grupo A, aunque en este caso las diferencias no son estadísticamente significativas $(\mathrm{gl}=1 ; \mathrm{F}=0,452 ; \mathrm{p}=* 0,502)$. En cuanto al $\mathrm{f} 2$, hay que señalar que es más alto para los informantes del grupo $\mathrm{B}$, aunque las diferencias no son estadísticamente significativas $(\mathrm{gl}=1 ; \mathrm{F}=$ $1,028 ; \mathrm{p}=* 0,311)$.

\begin{tabular}{|l|c|c|c|c|}
\hline & \multicolumn{2}{|c|}{ f1 } & \multicolumn{2}{c|}{ f2 } \\
\hline & A & B & A & B \\
\hline Media & 771,69 & 776,51 & 1600,65 & 1622,47 \\
\hline Sd & 89,91 & 85,59 & 241,82 & 283,57 \\
\hline
\end{tabular}

Según la provincia de origen de los informantes (tabla 5), la media del fl es más alta en Álava/Araba, seguida de Bizkaia, Gipuzkoa y Navarra; aunque las diferencias en algunos casos son muy pequeñas, son estadísticamente significativas $(\mathrm{gl}=3, \mathrm{~F}=10,279, \mathrm{p}=0,000)$. En el $\mathrm{f} 2$, la media más alta se da en Navarra, seguida de Bizkaia, Gipuzkoa y Álava/Araba. Las diferencias en este caso no son estadísticamente significativas $(\mathrm{gl}=3, \mathrm{~F}=1,856, \mathrm{p}=$ $* 0,136)$.

\begin{tabular}{|l|c|c|c|c|}
\hline & \multicolumn{2}{|c|}{ f1 } & \multicolumn{2}{c|}{ f2 } \\
\hline & Media & Sd & Media & Sd \\
\hline AR & 787,42 & 93,20 & 1545,92 & 290,41 \\
\hline BI & 785,92 & 86,01 & 1611,69 & 272,21 \\
\hline GI & 782,65 & 75,82 & 1609,35 & 253,71 \\
\hline NA & 739,61 & 92,10 & 1641,10 & 244,56 \\
\hline
\end{tabular}

Tabla 5: Medias y desviaciones del rango de f1 y f2 según la provincia de procedencia de los hablantes

En el gráfico de dispersión 4, podemos ver que entre las vocales de Bizkaia y Gipuzkoa las diferencias son muy pequeñas. Por el contrario, en Navarra la vocal es más alta y anterior que en el resto, y en Álava/Araba es la más posterior. 


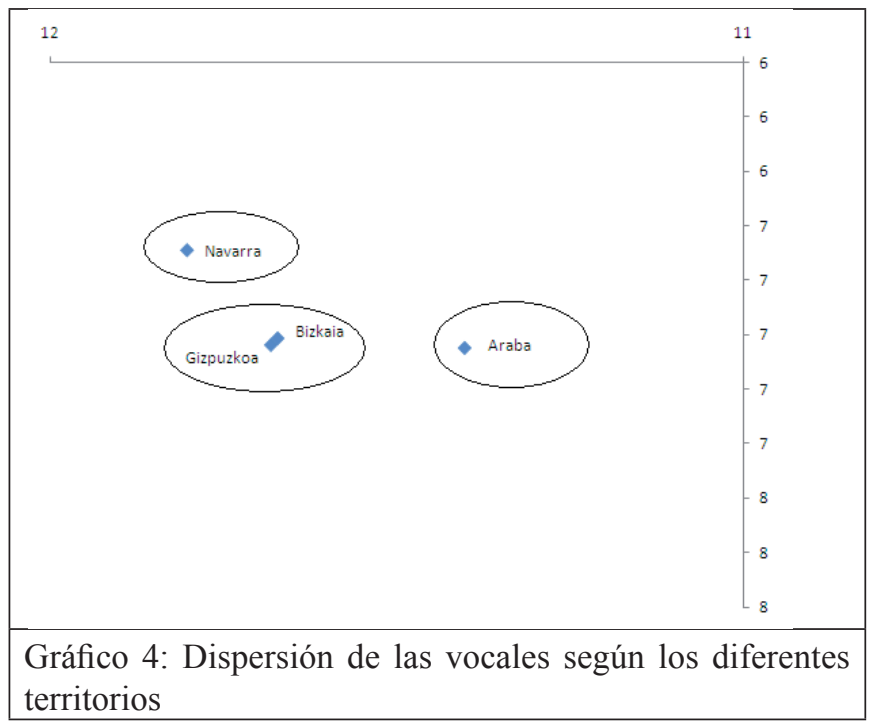

Por último, según el área sociolingüística de los informantes (tabla 6), la media del fl es más alta en el área 6, seguida por las áreas 2, 5, 1, 4, 7 y 3 ; siendo además las diferencias estadísticamente significativas $(\mathrm{gl}=6, \mathrm{~F}$ $=4,311, \mathrm{p}=0,000)$. En el f2, la media más alta se da en el área 6 , seguida de las áreas 7, 2, 4, 5, 3 y 1, aunque en este caso las diferencias no son estadísticamente significativas $(\mathrm{gl}=6, \mathrm{~F}=1,813, \mathrm{p}=* 0,094)$.

\begin{tabular}{|c|c|c|c|c|}
\hline & \multicolumn{2}{|c|}{ f1 } & \multicolumn{2}{c|}{ f2 } \\
\hline & Media & Sd & Media & Sd \\
\hline $\mathbf{( 1 )}$ & 777,67 & 80,69 & 1547,40 & 292,53 \\
\hline $\mathbf{( 2 )}$ & 796,63 & 77,83 & 1617,93 & 305,90 \\
\hline $\mathbf{( 3 )}$ & 732,36 & 43,74 & 1585,83 & 206,92 \\
\hline $\mathbf{( 4 )}$ & 771,40 & 89,20 & 1609,78 & 252,48 \\
\hline $\mathbf{( 5 )}$ & 779,34 & 94,77 & 1602,65 & 254,38 \\
\hline $\mathbf{( 6 )}$ & 800,00 & 70,29 & 1771,08 & 289,52 \\
\hline $\mathbf{( 7 )}$ & 738,50 & 95,79 & 1676,94 & 215,88 \\
\hline
\end{tabular}

Tabla 6: Medias y desviaciones del rango de f1 y f2 según las comarcas de procedencias de los hablantes

Como se muestra en el gráfico de dispersión 5, la menor diferencia se da en las áreas sociolingüísticas (2), (4) y (5), apareciendo el resto de las áreas más alejadas. 


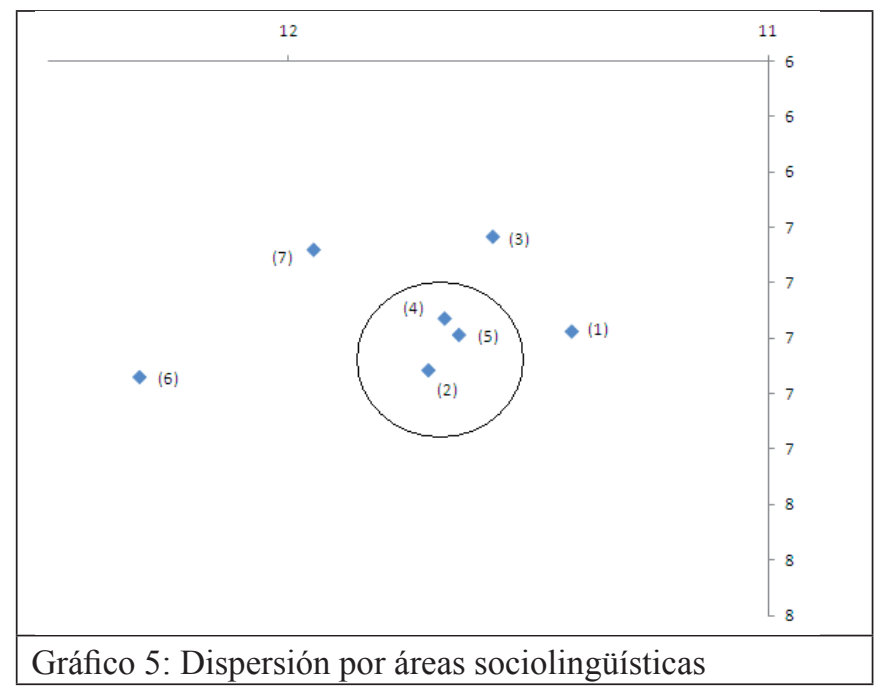

Los datos descritos hasta ahora revelan que el $\mathrm{f} 1$ se ve afectado estadísticamente por un número mayor de variables que el f2. Así, mientras al fl le afectan significativamente el tipo de emoción, la provincia de origen de los informantes y el área sociolingüística de pertenencia, al f2 solo le afecta el tipo de sílaba. En la tabla 7 mostramos el estadístico F y el nivel de significación para cada variable (p).

\begin{tabular}{|l|c|c|c|c|}
\hline & \multicolumn{2}{|c|}{ f1 } & \multicolumn{2}{c|}{ f2 } \\
\hline & F & p & F & p \\
\hline síl. & 1,431 & $* 0,233$ & 432,479 & 0,000 \\
\hline emo. & 12,785 & 0,000 & 1,544 & $* 0,214$ \\
\hline L.m. & 0,452 & $* 0,502$ & 1,028 & $* 0,311$ \\
\hline pro & 10,279 & 0,000 & 1,856 & $* 0,136$ \\
\hline á.soc. & 4,311 & 0,000 & 1,813 & $* 0,094$ \\
\hline
\end{tabular}

Tabla 7: Estadístico F y nivel de significación por cada variable

Según los datos obtenidos para el estadístico $\mathrm{F}$, la variable que más afecta al f1 es la emoción, seguida de la provincia de origen de los informantes y el área sociolingüística.

A continuación, analizaremos por separado los resultados de la variable 'emoción' junto con las variables 'provincia de origen de los informantes' y 'área sociolingüística de procedencia'. 
En la tabla 8, mostramos los resultados correspondientes a las variables 'emoción' y 'provincia de origen de los informantes'. Si se atiende al gráfico de dispersión 6, podemos apreciar tres áreas diferentes; en una se sitúan las vocales de Navarra, en otras las de Álava/Araba y en el centro las de Bizkaia y Gipuzkoa, muy próximas unas de otras.

\begin{tabular}{|l|c|c|c|c|c|}
\hline \multicolumn{2}{|c|}{} & \multicolumn{2}{|c|}{ f1 } & \multicolumn{2}{c|}{ f2 } \\
\hline Emo & Pro & Media & Sd. & Media & Sd. \\
\hline \multirow{4}{*}{ alegría } & AR & 819,15 & 101,33 & 1578,15 & 321,96 \\
\cline { 2 - 6 } & BI & 800,71 & 82,43 & 1634,30 & 270,76 \\
\cline { 2 - 6 } & GI & 795,69 & 69,19 & 1638,88 & 270,07 \\
\cline { 2 - 6 } & NA & 743,71 & 88,88 & 1639,04 & 240,45 \\
\hline \multirow{4}{*}{ tristeza } & AR & 752,95 & 75,09 & 1506,35 & 280,07 \\
\cline { 2 - 6 } & BI & 760,78 & 80,60 & 1578,26 & 255,75 \\
\cline { 2 - 6 } & GI & 765,29 & 74,07 & 1577,62 & 231,23 \\
\cline { 2 - 6 } & NA & 710,08 & 85,61 & 1641,08 & 258,14 \\
\hline \multirow{4}{*}{ enfado } & AR & 790,15 & 93,53 & 1553,25 & 277,48 \\
\cline { 2 - 6 } & BI & 796,26 & 90,10 & 1622,51 & 289,29 \\
\cline { 2 - 6 } & GI & 786,96 & 81,83 & 1611,54 & 259,42 \\
\cline { 2 - 6 } & NA & 765,04 & 94,94 & 1643,17 & 239,83 \\
\hline
\end{tabular}

Tabla 8: Medias y desviaciones de cada emoción atendiendo a la provincia de origen de los informantes

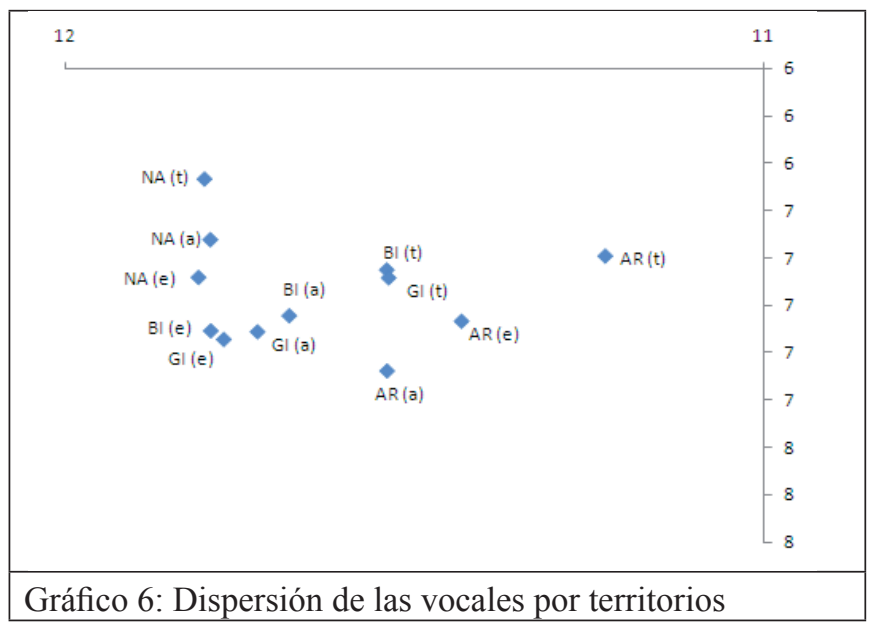


En la tabla 9, mostramos los resultados correspondientes a las variables de cada emoción según el área sociolingüística de pertenencia de los informantes. Si se observa el gráfico de dispersión 7, podemos distinguir una zona que se corresponde con el área (6), otra que se corresponde con el área (7) y una tercera, donde aparecen mezcladas las restantes áreas sociolingüísticas.

\begin{tabular}{|l|c|c|c|c|c|}
\hline \multicolumn{2}{|c|}{} & \multicolumn{2}{|c|}{ f1 } & \multicolumn{2}{c|}{ f2 } \\
\hline Emo & á. soc. & Media & Des. típ & Media & Des. típ \\
\hline alegría & $\mathbf{( 1 )}$ & 795,81 & 85,69 & 1595,44 & 334,97 \\
\cline { 2 - 6 } & $\mathbf{( 2 )}$ & 811,94 & 69,33 & 1636,08 & 291,07 \\
\cline { 2 - 6 } & $\mathbf{( 3 )}$ & 742,33 & 39,26 & 1606,58 & 219,29 \\
\cline { 2 - 6 } & $\mathbf{( 4 )}$ & 782,81 & 82,09 & 1628,22 & 260,63 \\
\cline { 2 - 6 } & $\mathbf{( 5 )}$ & 798,42 & 93,60 & 1620,27 & 266,05 \\
\cline { 2 - 6 } & $\mathbf{( 6 )}$ & 804,50 & 106,93 & 1839,25 & 284,21 \\
\cline { 2 - 6 } & $\mathbf{( 7 )}$ & 737,94 & 109,76 & 1667,56 & 219,47 \\
\hline tristeza & $\mathbf{( 1 )}$ & 735,50 & 69,10 & 1494,38 & 284,62 \\
\cline { 2 - 6 } & $\mathbf{( 2 )}$ & 768,44 & 77,31 & 1579,06 & 297,18 \\
\cline { 2 - 6 } & $\mathbf{( 3 )}$ & 713,83 & 47,44 & 1556,17 & 199,72 \\
\cline { 2 - 6 } & $\mathbf{( 4 )}$ & 737,28 & 88,06 & 1583,94 & 240,72 \\
\cline { 2 - 6 } & $\mathbf{( 5 )}$ & 759,94 & 85,49 & 1570,79 & 234,59 \\
\cline { 2 - 6 } & $\mathbf{( 6 )}$ & 808,00 & 45,51 & 1781,00 & 274,51 \\
\cline { 2 - 6 } & $\mathbf{( 7 )}$ & 741,69 & 84,95 & 1724,31 & 223,88 \\
\hline enfado & $\mathbf{( 1 )}$ & 801,69 & 73,62 & 1552,38 & 263,52 \\
\cline { 2 - 6 } & $\mathbf{( 2 )}$ & 809,50 & 80,68 & 1638,64 & 332,71 \\
\cline { 2 - 6 } & $\mathbf{( 3 )}$ & 740,92 & 41,61 & 1594,75 & 216,20 \\
\cline { 2 - 6 } & $\mathbf{( 4 )}$ & 794,09 & 88,26 & 1617,17 & 257,50 \\
\cline { 2 - 6 } & $\mathbf{( 5 )}$ & 779,65 & 102,33 & 1616,88 & 263,25 \\
\cline { 2 - 6 } & $\mathbf{( 6 )}$ & 787,50 & 65,49 & 1693,00 & 369,81 \\
\cline { 2 - 6 } & $\mathbf{( 7 )}$ & 735,88 & 97,29 & 1638,94 & 209,06 \\
\hline
\end{tabular}

Tabla 9: Medias y desviaciones de cada emoción según el área sociolingüística de los informantes 


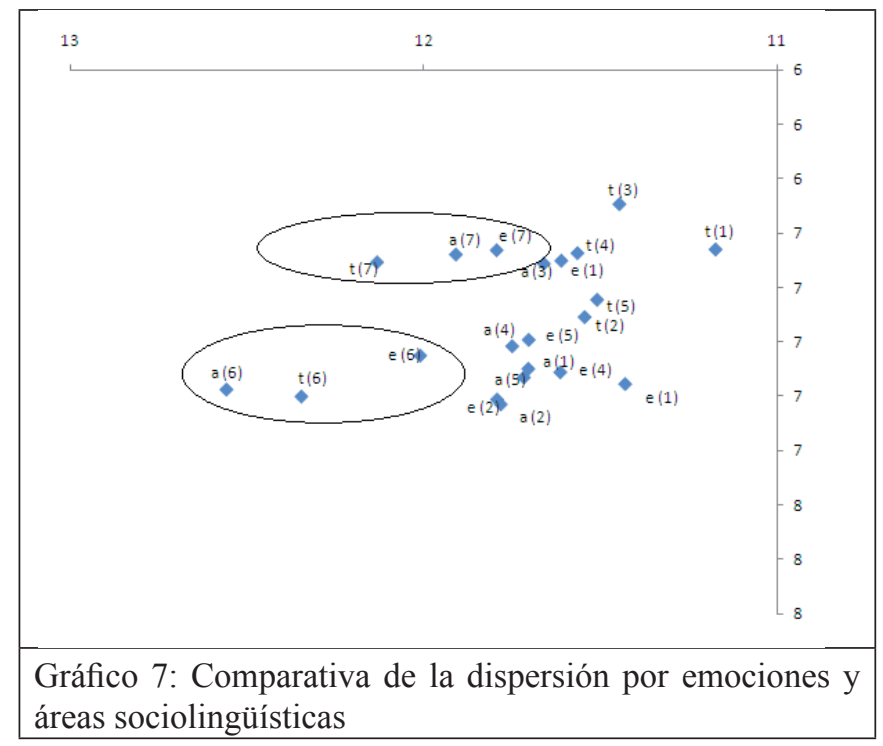

\section{CONCLUSIONES}

En esta sección pasamos a resumir las principales conclusiones que se pueden extraer del análisis de los datos presentados en la sección precedente.

En primer lugar, podemos concluir que de todas las variables analizadas y teniendo en cuenta aquellas estadísticamente significativas, el tipo de emoción de la frase es la variable que más afecta al fl, seguida de la provincia de origen de los informantes y el área sociolingüística de pertenencia de éstos. La media del f1 es superior en la emoción de alegría que en el enfado y la tristeza. En cuanto a la provincia de origen, la media de fl es más alta en Álava/Araba que en el resto; y por último, teniendo en cuenta el área sociolingüística a la que pertenecen los informantes, la media de f1 es más alta en la zona central de la provincia de Gipuzkoa, definida como área 6 en este trabajo, en la que se localizan las siguientes localidades: Aizarnazabal, Andoain, Elgoibar, Ordizia, Orio, Segura y Tolosa.

En cuanto al $\mathrm{f} 2$, la única variable que estadísticamente le afecta, es la pertenencia de la sílaba al sintagma anterior al verbo o al propio verbo. Cada sintagma tiene su propio espacio definido y dentro de éstos también cada 
sílaba. Las vocales del sintagma anterior al verbo son más anteriores y las del verbo más posteriores. En cuanto a las diferencias entre las emociones, solo la tristeza aparece claramente diferenciada en todos los casos, siendo ésta más abierta y posterior que la alegría y el enfado, que no presentan una separación clara entre ellas.

\section{REFERENCIAS BIBLIOGRÁFICAS}

Biemans, Monique. 2000. Gender variation in voice quality. Tesis doctoral, LOT Dissertation Series 38 .

Bobadilla, Jesús; Gómez, Pedro y Jesús Bernal. 1999. Posición y evolución de los formantes del habla. Estudios de Fonética Experimental 10: 11-40.

Boersma, Paul y David Weenink. 2009. Praat: doing phonetics by computer (Version 5.1) [Computer program]. [En línea]. Disponible en http://www.praat.org [Consulta $12 / 03 / 2015]$.

Etxebarria, Pilartxo. 1990. Zaldibiako bokalen azterketa akustikoa. Donostia: Mundaiz.

Etxebarria, Aintzane; Gaminde, Iñaki; Garay, Urtza y Asier Romero. 2014. Oinarrizko emozioen ezaugarri akustikoez. Fontes Linguae Vasconum. 46-117: 101-120.

Feraru, Silvia Monica. 2011. The comparisons between the formants values in French and Romanian. International Conference on Languages, E-Learning and Romanian Studies. [En línea].http://journals.lub.lu.se/index.php/elears/article/view/7015/5723[Consulta $12 / 03 / 2015]$.

Fernández Planas, Ana María. 2014. Las vocales, las glides y las consonantes sonantes en la cadena. En María Azucena Penas Ibáñez (ed.). Panorama de la fonética española actual, pp. 291-317. Madrid: Arco/Libros.

Gaminde, IÑaki. 2010. Bizkaiko Gazteen Prosodiaz: Euskaraz eta Gaztelaniaz. Bilbao: Mendebalde Kultura Alkartea eta Bizkaiko Foru Aldundia.

Gaminde, Iñaki; Etxebarria, Aintzane; Garay, Urtzay Asier Romero. 2013. Oinarrizko emozioen melodiez. Euskalingua 23: 14-27. [En línea].http://www.mendebalde.com/ modulos/usuariosFtp/conexion/archi573A.pdf. [Consulta 12/03/2015].

Gaminde, Iñaki; Etxebarria, Aintzane; Garay, UrtZa y Asier Romero. 2014a. Análisis fonológico de las emociones básicas simuladas en las variedades orientales de la lengua vasca. Dialectologia 12: 67-87.

Gaminde, Iñaki; Romero, Asier; Garay, Urtzay AintZane Etxebarria. 2014b. Análisis de las propiedades acústicas de las emociones básicas simuladas en bilingües precoces de vasco-español. Estudios de Fonética Experimental 23: 11-46.

GiL, Juana.1990. Los sonidos del lenguaje. Madrid: Síntesis.

Li, Aijun; Fang, Qiang; Hu, Fang; Zheng, Lu; Wang, Hong y Jianwu Dang. 2010. Acoustic and Articulatory Analysis on Mandarin Chinese Vowels in Emotional Speech. Report of Phonetic Research 10: 38-43.

Liscombe, Jackson J. 2007. Prosody and Speaker State: Paralinguistics, Pragmatics, and Proficiency. Tesis doctoral, Columbia University. 
Makarova, Veronika y Valery A. Petrushin. 2007. Sonorant segment quality in russian emotional speech. ICPHS-2007. [En línea].http://www.icphs2007.de/conference/ Papers/1316/1316.pdf[Consulta 12/03/2015].

Martínez Celdrán, Eugenio. 1989. Fonética. Barcelona: Teide.

Navas, Eva; Inmaculada Hernáez e Iker Luengo. 2006. An Objective and Subjective Study of the Role of Semantics and Prosodic Features in Building Corpora for Emotional TTS. IEEE Transactions on audio, speech and language processing 14-4: 1117-1127.

Navas, Eva; Inmaculada Hernáez; Iker Luengo; IÑaki Sainz; Ibon Saratxaga y Jon Sanchez. 2007. Meaningful Parameters in Emotion Characterisation. LNAI4775: 74-84

PribiL, JIŘí Y AnNA PřIBILOVÁ. 2013. Determination of Formant Features in Czech and Slovak for GMM Emotional Speech Classifier. Radioengineering 22-1: 52-59.

Quilis, Antonio. 1988. Fonética acústica de la lengua española. Madrid: Gredos.

Sainz, Iñaki; Ibon SaratXaga; Eva Navas; Inmaculada Hernáez; Jon SAnchez; Iker Luengo; Igor Odriozola y Eneritz de Bilbao. 2008. Evaluación Subjetiva de una Base de Datos de Habla Emocional para Euskera. V Jornadas en Tecnología del Habla, pp. 191-194.

Schötz, Susanne. 2006. Perception, Analysis and Synthesis of Speaker Age. Tesis doctoral. Lund University. [En línea].http://person.sol.lu.se/SusanneSchotz/downloads/Thesis_S_ Schoetz_2006.pdf [Consulta 12/03/2015].

SoziolinguistiKa Klusterra. 2013. Hizkuntzen egoera soziala Euskal Herrian. Datu biltegia. [En línea].http://www.soziolinguistika.org/edb [Consulta 12/03/2015].

Urrutia, Hernan; Etxebarria, Maitane; Turrez, Itziar y Álex Iribar. 1995. Fonética vasca 4 (Las vocales en euskera). Bilbao:Universidad de Deusto.

Yildirim, Serdar; Murtaza Bulut; Chul Min Lee; Abe Kazemzadeh; Carlos Busso; Zhigang Deng; Sungbok Lee y Shrikanth Narayanan. 2004. An acoustic study of emotions expressed in speech. En W.J. Barry (ed.). 8th International Conference on Spoken Language Processing, pp. 2193-2196. Jeju Island, Korea: ISCA.

ZuAzo, Koldo. 1998. Euskalkiak gaur. Fontes Linguae Vasconum 78: 191-234. 